\section{Uninformed Individuals Promote Democratic Consensus in Animal Groups}

lain D. Couzin, ${ }^{{ }^{*}}$ Christos C. Ioannou, ${ }^{1} \dagger$ Güven Demirel, ${ }^{2}$ Thilo Gross, ${ }^{2} \ddagger$ Colin $]^{\text {. Torney, }}{ }^{1}$ Andrew Hartnett, ${ }^{1}$ Larissa Conradt, ${ }^{3} \S$ Simon A. Levin, ${ }^{1}$ Naomi E. Leonard ${ }^{4}$

Conflicting interests among group members are common when making collective decisions, yet failure to achieve consensus can be costly. Under these circumstances individuals may be susceptible to manipulation by a strongly opinionated, or extremist, minority. It has previously been argued, for humans and animals, that social groups containing individuals who are uninformed, or exhibit weak preferences, are particularly vulnerable to such manipulative agents. Here, we use theory and experiment to demonstrate that, for a wide range of conditions, a strongly opinionated minority can dictate group choice, but the presence of uninformed individuals spontaneously inhibits this process, returning control to the numerical majority. Our results emphasize the role of uninformed individuals in achieving democratic consensus amid internal group conflict and informational constraints.

$\mathrm{S}$ ocial organisms must often achieve a consensus to obtain the benefits of group living and to avoid the costs of indecision ( 1 12). In some societies, notably those of eu social insects, making consensus decisions is often a unitary, conflict free process because the close relatedness among individuals means that they typically share preferences $(1 I)$. However, in other social animals, such as schooling fish, flocking birds, herding ungulates, and humans, individual group members may be of low relatedness; thus, self interest can play an important role in group decisions. Reaching a consensus decision, there fore, frequently depends on individuals resolving complex conflicts of interest $(111,13,14)$.

There are several means of achieving group consensus. In some cases, decisions made by one or only a small proportion of the group dictate the behavior of the entire group $(46,13,14)$. There fore, a minority, or even a single individual, has the potential to control or exploit the majority, achieving substantial gains at the expense of other group members (1 $6,9,10,14)$. In contrast, consensus can also be reached through demo cratic means, with fair representation and an out come determined by a plurality. Democratic decisions tend to be more moderate, minimiz ing group consensus costs, particularly in large animal groups (3). However, in the absence of established procedures such as voting $(8)$, it is unclear how equal representation is enforced.

${ }^{1}$ Department of Ecology and Evolutionary Biology, Princeton University, Princeton, N] 08544, USA. ${ }^{2}$ Max Planck Institute for Physics of Complex Systems, Nöthnitzer Strasse, 01187 Dresden, Germany. ${ }^{3}$ School of life Sciences, John Maynard Smith Build ing, University of Sussex, Falmer, Brighton BN1 9QG, UK. ${ }^{4} \mathrm{De}$ partment of Mechanical and Aerospace Engineering, Princeton University, Princeton, N] 08544, USA.

*To whom correspondence should be addressed. E mail: icouzin@princeton.edu

†Present address: School of Biological Sciences, University of Bristol, Woodland Road, Bristol BS8 1UG, UK. †Present address: Merchant Venturers School of Engineer ing, University of Bristol, Bristol BS8 1UB, UK. §Present address: Department of Zoology, Downing Street, University of Cambridge, Cambridge CB2 3EU, UK.
Consequently, for both human societies $(1,2,6,9,10,14)$ and group living animals $(6,13)$, it has been argued that group decisions can be subject to manipulation by a self interested and opinionated minority. In particular, previous work suggests that groups containing individu als who are uninformed, or naïve, about the de cision being made are particularly vulnerable to such manipulation $(2,9,10,13)$. Under this view, uninformed individuals destabilize the capacity for collective intelligence in groups $(10,14)$, with poorly informed individuals potentially facilitat ing the establishment of extremist opinions in populations $(9,14)$.

Here, we address the question of whether and, if so, under which conditions a self interested and strongly opinionated minority can exert its influence on group movement decisions. We show

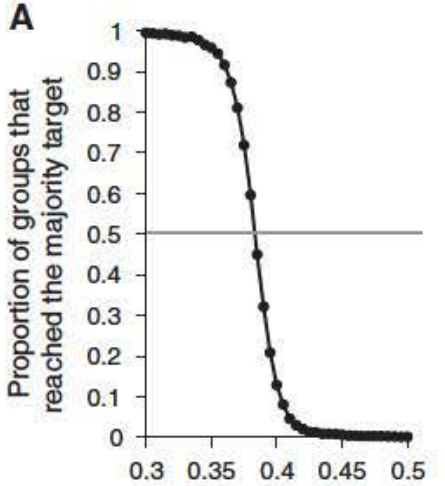

Strength of minority preference, $\omega_{2}$ infrequent, see fig. S5). $\omega_{1}=0.3$. See (15) for details. that uninformed individuals (defined as those who lack a preference or are uninformed about the features on which the collective decision is being made) play a central role in achieving dem ocratic consensus.

We use a spatially explicit computational model of animal groups (15) that makes minimal assumptions regarding the capabilities of indi vidual group members; they are assumed to avoid collisions with others and otherwise exhibit the capacity to be attracted toward, and to align direction of travel with, near neighbors $(5,16)$. We investigate the case of consensus decision making regarding a choice to move to one of two discrete targets in space (thus, the options are mutually exclusive).

The direction and strength of an individual's preference are encoded in a vector term $\overrightarrow{\boldsymbol{\omega}}$ (di rected toward the individual's preferred target). Higher scalar values of $\omega$ (equivalent to the length of the $\vec{\omega}$ vector, $\omega \equiv|\overrightarrow{\boldsymbol{\omega}}|$ ) represent a greater conviction in, or strength of, individual preference to move in the direction of the target and, thus, also represent greater intransigence to social in fluence (5).We explore the case where there are two subpopulations within the group $N_{1}$ and $\mathrm{N}_{2}$, respectively that have different preferred targets. Because we are interested in determining whether a minority can exploit a majority, we set $N_{1}>N_{2}$ for the simulation. The strengths of the preference of the numerical majority and minor ity are represented by their respective $\omega$ values, $\omega_{1}$ and $\omega_{2}$. See (15) for details.

If the strength of the majority preference $\left(\omega_{1}\right)$ is equal to or stronger than the minority pref erence $\left(\omega_{2}\right)$, the group has a high probability of reaching the majority preferred target (Fig. 1A) (5). Yet increasing $\omega_{2}$ (beyond $\omega_{1}$ ) can result

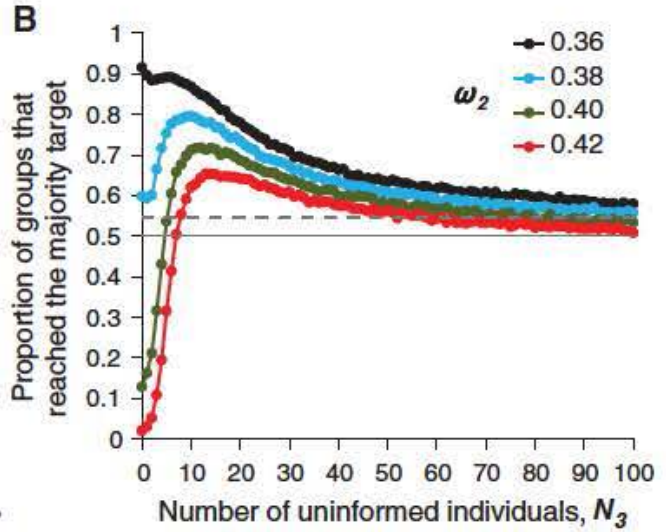

Fig. 1. Spatial simulation of consensus decision-making in which individuals' preferred direction, weighted by their respective $\omega$ (see main text), is directed toward their preferred target. (A) $\omega_{1}=0.3$. All individuals are informed with majority $\boldsymbol{N}_{1}=6$ and minority $\boldsymbol{N}_{2}=5$. As the minority increases its preference strength, $\omega_{2}$, it increasingly controls group motion. (B) In the presence of sufficient uninformed individuals, the minority can no longer exploit the majority by increasing $\omega_{2}$ (see fig. S2 for other values of $\boldsymbol{N}_{1}$ and $\left.\boldsymbol{N}_{2}\right)$. The ratio of the majority to all informed, $\boldsymbol{N}_{\mathbf{1}} /\left(\boldsymbol{N}_{\mathbf{1}}+\boldsymbol{N}_{2}\right)$, is shown as a horizontal gray dashed line. The proportion reaching the majority target is calculated as the number of times (from 20,000 replicates) the majority-preferred target is reached divided by the number of times a (minority or majority) target was reached (i.e., only consensus decisions were evaluated; splitting was 
Fig. 2. The adaptive-network model $(17,21-23)$ provides an analytically tractable analog to the spatially explicit model. (A) Network simulations show qualitative agreement with the spatial model. (B) An analytical approximation of the model reveals the dynamical cause of this transition. For low densities of uninformed individuals, the minoritycontrolled state (red line) is the only stable attractor. As the proportion of uninformed individuals increases, the system undergoes a saddle-node bifurcation resulting in a stable majoritycontrolled state (solid black line) and an unstable undecided state (dashed black line). Simulations (blue circles) closely match analytical approximations. (C) In this phase diagram, we see the outcome of opinion formation as a function of the ratio of the majority to minority subpopulation $\left(\boldsymbol{N}_{1} / N_{2}\right)$ and the relative strength of the minority preference (equivalent to $\omega_{2} / \omega_{1}$ ) measured in terms of the ratio of switching rates [see (15) for details]. The white region of the phase
B

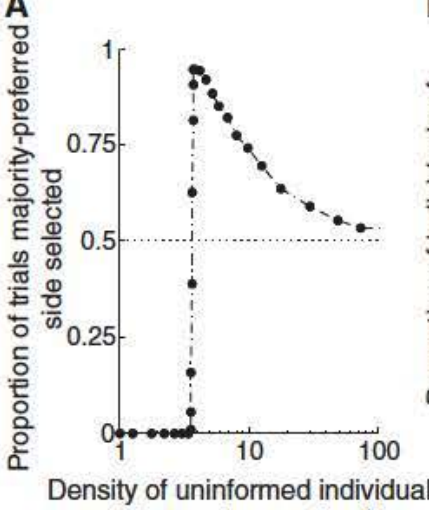

(informed proportion ${ }^{-1}$ )

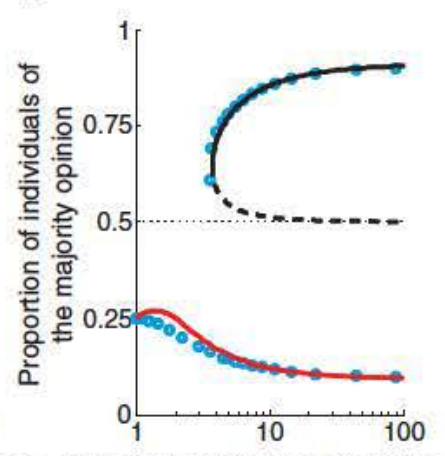

Density of uninformed individuals (informed proportion ${ }^{-1}$ )
C

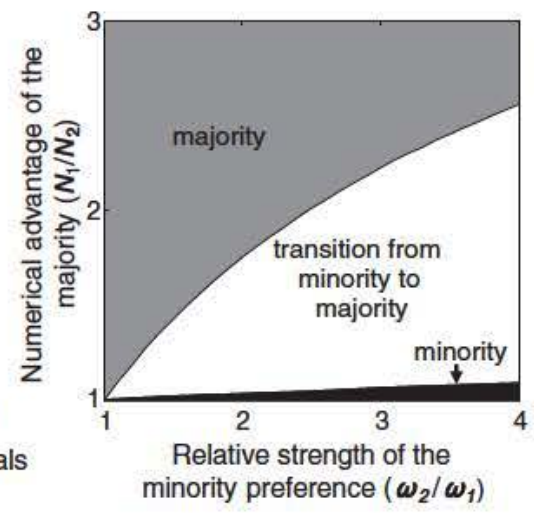

diagram represents the region in which this saddle-node bifurcation results in a transition from minority to majority control when sufficient uninformed individuals are present. The regions denoted "majority" and "minority" represent parameter space where the $\boldsymbol{N}_{\mathbf{1}}$ or $\boldsymbol{N}_{\mathbf{2}}$ preferences, respectively, are adopted regardless of the number of uninformed individuals $\left(\boldsymbol{W}_{3} \in[0, \infty]\right)$.

in the minority gaining control and eventually dictating group outcome (Fig. 1A and fig. S1). If some individuals do not have relevant prior information or are only weakly biased, however, as is likely in many animal groups $(5,6,13)$, then groups can be considered to have a third sub population of $N_{3}$ individuals with $\omega_{3} \approx 0$. Now when $\omega_{2}$ is in the range where the minority dictates the group outcome for $N_{3} \quad 0$, adding uninformed individuals tends to return control spontaneously to the numerical majority (Fig. 1B) $\left(\omega_{2} \quad 0.4,0.42\right)$. As $N_{3}$ increases, this effect reaches a maximum and then begins to slowly diminish. Eventually, noise dominates and un informed individuals neither amplify a weak nu merical majority nor lend substantial support to the minority.

To determine whether these results can be generalized, we develop reduced, analytically tractable versions of the above model. The first, modified from (17), represents individuals as nodes on a network with interindividual commu nication represented by a dynamically changing edge topology. A second (increasingly mini malist) approach considers a convention game of self reinforcing normative opinion dynamics (18). These simplified models are nonspatial and consider discrete (binary) opinions, yet incorpo rate key features of the spatial model: (i) Indi viduals adopt, probabilistically, the opinion they perceive to be that of the local majority (this results in positive feedback reinforcing the pre dominant opinion and, consequently, rapid non linear transitions from disordered to ordered consensus states). (ii) The strength of individ ual preference manifests as intransigence during interactions with others.

These models capture the same qualitative collective features as the spatial model (15). Figure $2 \mathrm{~A}$ shows the presence of a sharp tran sition from a minority to majority controlled outcome in the network model as the density of

Fig. 3. Experiments with schooling fish demonstrate support for our hypothesis. (A) When the minority $\left(W_{2}=5\right)$ are trained to the intrinsically preferred (yellow) target, inclusion of untrained individuals returns control from a dominating minority to the numerical majority (18 replicates per data point). (Inset) A golden shiner is shown. (B) Image from an experimental video with $\boldsymbol{N}_{\mathbf{1}}=6, \boldsymbol{N}_{2}=5$, and $\boldsymbol{N}_{\mathbf{3}}=$ 10. See fig. S3 and (15) for further details.
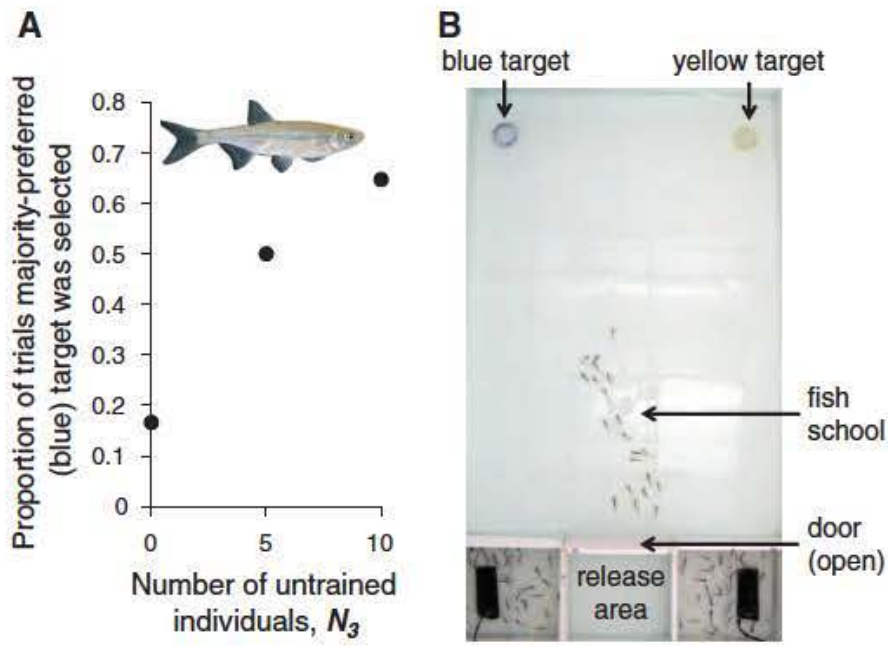

uninformed individuals is increased. Analysis reveals the dynamical nature of this transition (Fig. 2B) (15), as well as the large region of parameter space in which a minority preferred outcome switches to a majority preferred outcome if sufficient uninformed individuals are present (white region in Fig. 2C).

In all models, an entrenched minority is ca pable of exerting substantial influence by biasing the perceived consensus. Because they exhibit little intransigence or intrinsic bias, however, un informed individuals will lend support to, and tend to amplify, a numerical advantage (even a slight one). If sufficiently numerous, they reduce the effect of intransigence and inhibit the capacity for the minority to take hold, thus returning con trol to the numerical majority. Consequently, even a small change in the number of uninformed individuals can dramatically alter the outcome of consensus decisions (Figs. 1B and 2A and figs. S7A and S8) (15). We emphasize that this pro cess will tend to inhibit any strong minority preference, regardless of the intrinsic quality or value of that view. We conjecture that this phe nomenon may be found in seemingly disparate systems that share those common features out lined above (15).

Our theoretical studies make a primary test able prediction: Uninformed individuals should inhibit the influence of a strongly opinionated minority, retuming control to the numerical ma jority. To test this prediction, we conducted ex periments with golden shiners (Notemigonus crysoleucas) (Fig. 3A, inset), a strongly school ing species of freshwater fish (19). We trained two subpopulations of individuals (representing either $N_{1}$ or $N_{2}$ ) to have preferences to move from a starting location toward either a blue target or a yellow target (Fig. 3B and figs. S3 and S4) (15). Under our experimental conditions, shiners exhibited a spontaneous preexisting bias toward the yellow target $(15,20)$, evident in both training (figs. S10 and S11) and testing (see results, below). Consequently, we did not need to 
employ different training regimes to create a difference in the strength of preference between our two trained subpopulations (15). A third $\left(\boldsymbol{N}_{\mathbf{3}}\right)$ subpopulation was left untrained.

Because our theoretical predictions do not depend on the absolute number of $\boldsymbol{N}_{\mathbf{1}}$ individuals (fig. S2), and due to the time consuming nature of training and constraints related to obtaining enough fish for replication, we set $N_{1} \quad 6$ and $\mathrm{N}_{2} 5$ fish (as in Fig. 1). Our simulations also predict a large effect for a relatively small number of naïve individuals (Fig. 1B); thus, we set $\boldsymbol{N}_{\mathbf{3}}$ 0,5 , or 10 . When $\boldsymbol{N}_{2}$ fish are trained to the yellow (biased) target and all individuals exhibit a preference $\left(\begin{array}{ll}\boldsymbol{N}_{\mathbf{3}} & 0\end{array}\right)$, the minority $\boldsymbol{N}_{\mathbf{2}}$ dictates the consensus achieved, even though the fish trained to the blue target are more numerous. However, when untrained individuals are present, they increasingly return control to the numerical ma jority $N_{\mathbf{1}}$ (Fig. 3A) [generalized linear mode (GLM); likelihood ratio test (LRT) $)_{1,52} \quad 5.60, P$ 0.018]. A snapshot from a trial is shown in Fig. 3B. We also performed experiments in which individuals with the stronger preference were also in the numerical majority $\left(N_{1}\right.$ trained to the yellow target). As expected (15), the majority was more likely to win ( $72 \%$ of trials overall), and the presence of uninformed individuals had no effect $\left(12,16\right.$, and 11 of 18 replicates for $N_{3}$ 0,5 , and 10, respectively; GLM; $\operatorname{LRT}_{1,52} \quad 0.14$, $P \quad 0.71)$.

Our work provides evidence that uninformed individuals play an important role in consensus decision making: By enforcing equal representa tion of preferences in a group, they promote a democratic outcome. This provides a new under standing of how informational status influences consensus decisions and why consensus decision making may be so widespread in nature (4). Fur thermore, these results suggest a principle that may extend to self organized decisions among hu man agents.

\section{References and Notes}

1. K. Arrow, Social Choices and Individual Values (Yale Univ. Press, New Haven, CT, ed. 2, 1963).

2. J. M. Buchanan, G. Tullock, The Calculus of Consent: Logical Foundations of Constitutional Democracy (Liberty Fund, Indianapolis, IN, 1958).

3. L. Conradt, T. J. Roper, Nature 421, 155 (2003).

4. L. Conradt, T. J. Roper, Trends Ecol. Evol. 20, 449 (2005).

5. I. D. Couzin, J. Krause, N. R. Franks, S. A. Levin, Nature 433, 513 (2005).

6. A. J. King, D. D. P. Johnson, M. Van Vugt, Curr. Biol. 19, R911 (2009).

7. J. Krause, G. D. Ruxton, Living in Groups (Oxford Univ. Press, Oxford, 2002)

8. J. Mansbridge, Beyond Adversary Democracy (The Univ. of Chicago Press, Chicago, 1983).

9. M. Olson Jr., The Logic of Collective Action: Public Goods and the Theory of Groups (Harvard Univ. Press, Cambridge, MA, 1971).

10. W. Riker, Liberalism Against Populism: A Confrontation Between the Theory of Democracy and the Theory of Social Choice (W. H. Freeman, San Francisco, 1982).

11. T. D. Seeley, Honeybee Democracy (Princeton Univ. Press, Princeton, N], 2010).

12. A. J. Ward, J. E. Herbert Read, D. J. T. Sumpter, J. Krause, Proc. Natl. Acad. Sci. U.S.A. 108, 2312 (2011).

13. L. Conradt, J. Krause, I. D. Couzin, T. J. Roper, Am. Nat. 173, 304 (2009).

14. S. Issacharoff, Int. J. Const. Law 6, 231 (2008).
15. Materials and methods are available as supporting material on Science Online.

16. Y. Katz, K. Tunstrøm, C. C. Ioannou, C. Huepe, I. D. Couzin, Proc. Natl. Acad. Sci. U.S.A. 108, 18720 (2011).

17. C. Huepe, G. Zschaler, A. L. Do, T. Gross, New J. Phys. 13 073022 (2011)

18. H. P. Young, Individual Strategy and Social Structure: An Evolutionary Theory of Institutions (Princeton Univ. Press, Princeton, N], 1998).

19. S. G. Reebs, Anim. Behav. 59, 403 (2000).

20. R. Spence, R. Smith, Ethology 114, 582 (2008).

21. P. Holme, M. E. J. Newman, Phys. Rev. E. 74, 056108 (2006)

22. F. Vazquez, V. M. Eguíluz, M. San Miguel, Phys. Rev. Lett. 100, 108702 (2008).

23. D. H. Zanette, S. Gil, Physica D 224, 156 (2006).

Acknowledgments: We thank H. Li, L. Petzold, J. Moehlis, A. Kolpas, Y. Katz, and A. Kao for help with CUDA and the Couzin lab for discussions. This work was supported by NSF grant PHY 0848755, the Searle Scholars Program, Office of Naval Research grant N00014 091 1074, Defense Advanced Research Projects Agency grant HR0011 091 0055, and The Royal Society. I.D.C. devised the study and performed and analyzed the spatial simulations, C.C.I. performed and analyzed the fish experiments, and G.D. and T.G. performed the adaptive networks model and C.J.T. the convention model. All authors participated in some aspects of model formulation and analysis. I.D.C. wrote the paper with input from all authors. Data and code freely available at http://icouzin. princeton.edu/uninformed individuals promote democratic consensus in animal groups/. 